\title{
Comparative Analysis and Detection of Brain Tumor Using Fusion Technique of T1 and T2 Weighted MR Images
}

Padmanjali A. Hagargi, SVERIs COE, Pandharpur, India

\begin{abstract}
Image fusion is a technique to fuse the two or more images. As the fused image gathers more information as comparative to the single image, image fusion of multiple images can be done to extract more number of information, with this reason the it is important in the field of medical image analysis. The fusion technique is so useful in detection of different kind of disease using different kind of medical images. Brain tumor disease is a large issue because of non-proper diagnosis and treatment is lacking accordingly. Using T1, T2 Weighted MR images are two medical MR images at different time constant during the scanning of brain tumor. These two or more images can be used to extract more information by the various image fusion technique.
\end{abstract}

\section{KEYWORDS}

Art Classifier, Brain Tumor, Curvelet Transforms, Discrete Wavelet Transforms, Feature Extraction, Fusion Technique, Medical MRI, PSNR, RMS, T1, T2, Weighted MRI

\section{INTRODUCTION}

The fusion technique involves integrating or combining the two or more level information together to obtain more accurate information. Here in our proposed method before fusing two MRI images, the process of reducing the noise elements in the source images is done using Weiner Filter and then two-level fusion is performed. Discrete wavelet transforms (DWT) and curvelet transforms (CT) (Candes \& Donoho 2000) are used for hybrid fusion. Fusion of images is done using co-efficient developed. DWT Coefficients will be of two levels; detailed and approximate coefficient. Approximate coefficients are applied further to Curvelet Transform and detailed coefficient is directly assigned to fusion rule. Resulted coefficients are combined with detailed coefficients developed by DWT(Candes \& Donoho 2005) using fusion rule.

\subsection{Fusion of Brain Tumor MRI using DWT and CT Techniques}

\subsubsection{Curvelet}

Curvelet(Starck et al., 2002) will develop low-low, low-high, high-low and high-high bands with the information of the image. Among all four low-low band consists of highest information of the 
coefficient. Ignoring rest three bands fusion rules are applied only on the low-low band. Once the fusion rule is applied coefficients are to be converted back to their original spatial domain. Inverse transforms are applied on fused values to rebuilt spatial domain values that can be analyzed easily. The final fused image represents the image with the information integrating into it of both two input images.

\subsubsection{DWT}

In numerical analysis and functional analysis, a discrete wavelet transform (DWT)(Sruthy et al., 2013) is any wavelet transform for which the wavelets are discretely sampled. As with other wavelet transforms, a key advantage it has over Fourier transforms is temporal resolution: it captures other frequency and location information (location in time). The Figure 1 shows how to extract the approximate coefficients and detailed coefficients and these information are further fused using simple average fusion rule respectively for both the coefficient to extract features of fused coefficients of information.

\section{FUSION OF T1, T2 WEIGHTED MRI USING RPCA AND QUADTREE TECHNIQUES}

In Previous fusion method, image fusion is done using curvelet and DWT. In this fusion Fuzzy based fusion is done on the coefficients generated by RPCA(Wright et al., 2009) (Robust Principal Component Analysis) and Quadtree based decomposition techniques.

Figure 1. Fusion using feature extraction

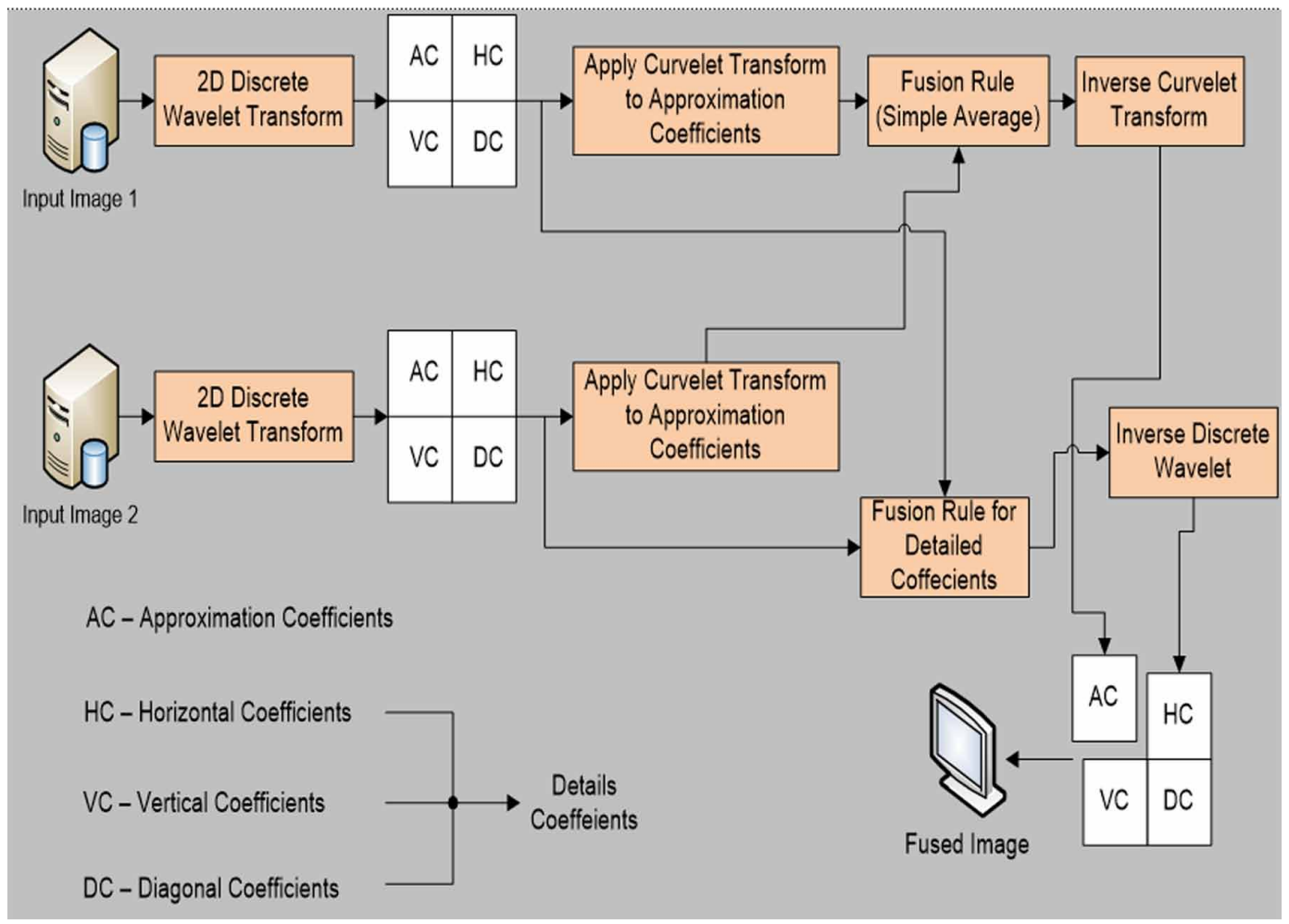


In this method, two weighted image are preprocessed using wiener filter and then fused using RPCA and Quadtree decomposition. After fusion of RPCA and Quadtree decomposition method the resulted fused image is compared with the images fused using DWT and CT. The comparison is taken by considering two parameters: PSNR and RMSE. The method with better parametric values will be considered as the best fusion method in our proposed method. The best out of these two methods will be considered for the segmentation stage. In the segmentation stage, tumor parts in the fused image will be segmented out using level set segmentation. The resulted image of segmentation stage is used as an input for the phase 3 for the features calculations and classification.

\subsection{RPCA}

Robust Principal Component Analysis (RPCA)(Wright et al., 2009) is a modification of the widely used statistical procedure of Principle Component Analysis (PCA) which works well with respect to grossly corrupted observations. A number of different approaches exist for Robust PCA, including an idealized version of Robust PCA, which aims to recover a low-rank matrix $\mathrm{L}_{0}$ from highly corrupted measurements $\mathrm{M}=\mathrm{L}_{0}+\mathrm{S}_{0}$. This decomposition in low-rank and sparse matrices can be achieved by techniques such as Principal Component Pursuit method (PCP), Stable PCP, Quantized PCP, Block based PCP and Local PCP Then, optimization methods are used such as the Augmented Language Multiplier, Alternating Detection Method (ADM), Fast Alternating Minimization (FAM) or Iteratively Reweighted Least Squares (IRLS).

\subsection{Quadtree}

A quadtree is a tree data structure in which each internal node has exactly four children. Quadtrees are the two-dimensional analog of Octrees and are most often used to partition a two-dimensional space by recursively subdividing it into four quadrants or regions. The data associated with a leaf cell varies by application, but the leaf cell represents a "unit of interesting spatial information". The subdivided regions may be square or rectangular, or may have arbitrary shapes. A similar partitioning is also known as a Q-tree decomposition. All forms of quadtrees share some common features: They decompose space into adaptable cells Each cell (or bucket) has a maximum capacity. When maximum capacity is reached, the bucket splits The tree directory follows the spatial decomposition of the quadtree. The Figure 2 shows the fusion of T1 and T2 Weighted MR images are fused using RPCA and QT decomposition respectively and their performance results are compared among both the fusion techniques, to detect the tumor segmented sections.

RPCA generates two sparse matrixes $\mathrm{S}_{\mathrm{m} 1}$ and $\mathrm{S}_{\mathrm{m} 2}$ each for each image. This two matrices are combined into single sparse matrix $S_{m}$ and then passed to Quadtree decomposition module. Decomposition is performed on the sparse matrix values by Quadtree decomposition. In our method EOG (Energy of image gradient) has used to keep track on homogeneity information. $\mathrm{EOG}_{\mathrm{A}}$ and $\mathrm{EOG}_{\mathrm{B}}$ are the two EOG's generated for T1-T2 images respectively. The calculations done for active levels by EOG are given to decision matrix. These decomposed values are then filtered out before going for the fusion rule. The method utilized by us for the fusion is fuzzy. Fuzzy being the module of rules has its own criterion in the image processing field. Pixels to pixel level calculations are allowed by this method.

\section{GLCM AND GLRLM FEATURE EXTRACTION AND FUSION}

In this fusion is divided into two phase training and testing phase. In training phase the tumor part of segmented brain image is taken as input and its features are extracted using GLCM (Gray Scale Co-occurrence Matrix) and GLRLM (Grey-level run-length matrix). The extracted features of tumor part are stored in knowledge base. Similarly at the testing side two separate Brain images are preprocessed using Weiner filter, fused using RPCA and Quadtree decomposition and then segmented 


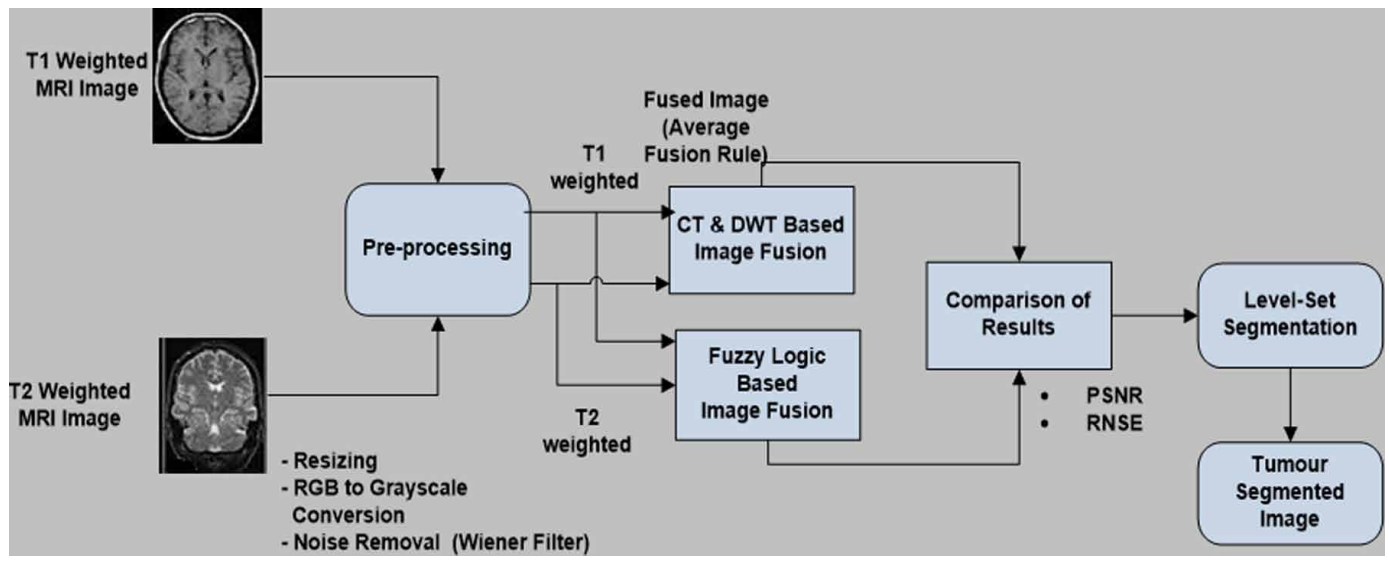

using Level set Segmentation (Jaffar et al., 2011; Mustaqeem et al., 2012). The segmented tumor part is taken out and features of that image are extracted. Finally ANN (Artificial Neural Network) classifier is used to classify the tested and trained features. ANN classifies features either benign or malignant. The proposed system is mainly concentrated to classify the given tumor stage is accurately.

The performance of the designed system is measured with a set of trained and tested samples, the application of confusion matrix can effectively give information about True Positive, True Negative, False Positive and False Negative samples. The proposed system present the result with accuracy is 93.45\%. In next phase we mainly concentrated to improve the accuracy level. The advanced features extraction techniques and machine learning classifiers are used to improve the performance level. The Figure 3 shows the image fusion using the RPCA and QT decomposition to get the tumor segmented sections and further the features are extracted using GLCM and GLRLM, and further using ANN classifier are classified as tumor and non-tumor.

\section{CLBP AND PHOG FEATURE EXTRACTION AND FUSION}

This method is divided into two phase training and testing phase. In training phase the tumor part of segmented brain image is taken as input and its features are extracted using Complete Local Binary Pattern (CLBP)(Sinha et al., 2012) and Pyramid Histogram of Orientation Gradients (PHOG)(Sinha et al., 2012). The extracted features of tumor part are stored in knowledge base. Similarly at the testing side two separate Brain images are pre-processed using Weiner filter, fused using RPCA and Quadtree decomposition and then segmented using Level set Segmentation(Mahantesh \& Kanyakumari 2016). The segmented tumor(Samriti \& Singh 2016) part is taken out and features of that image are extracted. Finally ART classifier is used to classify the tested and trained features. ART (Adaptive Resonance Theory) classifies features either benign or malignant. The performance of the proposed system is analysed by using confusion matrix. The application advanced feature extraction algorithm and machine leaning classifier can effectively increases the performance level i.e. $97.3 \%$. In phase 4 there is an improvement in performance accuracy (i.e. as compared to phase 3 ).

\subsection{Complete Local Binary Patterns (LBP)}

This is a type of visual descriptor used for classification in Computer Vision. LBP is the particular case of the Texture Spectrum model proposed in 1990 LBP was first described in 1994. It has since been found to be a powerful feature for texture classification; it has further been determined that when CLBP(Tapia et al., 2010) is combined with the Histogram of Oriented Gradients (HOG) descriptor, 
Figure 3. Architecture of train and testing using GLCM and GLRLM

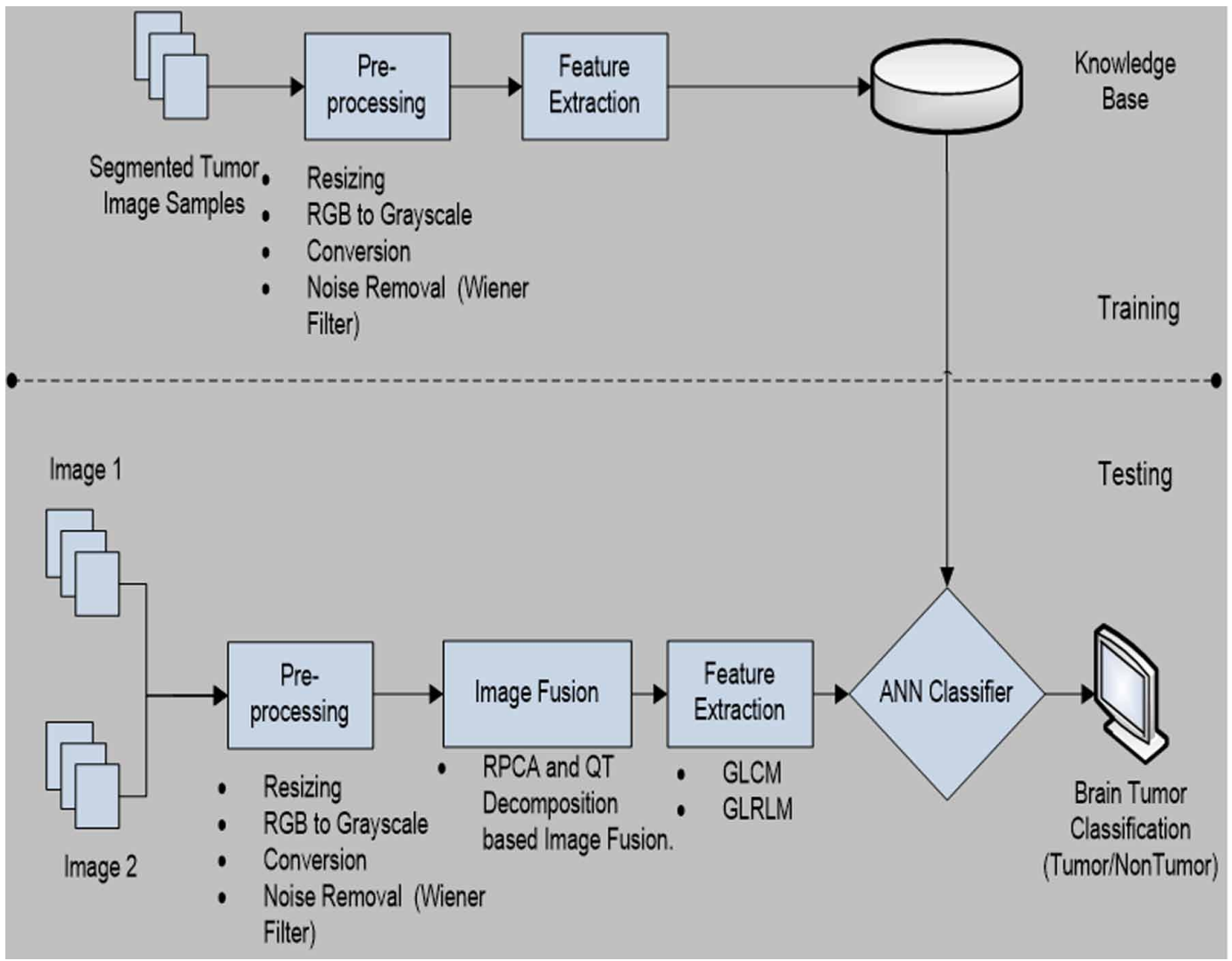

it improves the detection performance considerably on some datasets. A comparison of several improvements of the original LBP in the field of background subtraction was made in 2015 by Silva et al. A full survey of the different versions of LBP can be found in Bouwman. The Figure 4 shows the image fusion using the RPCA and QT decomposition to get the tumor segmented sections and further the features are extracted using CLBP and PHOG, and further using ART classifier are classified as tumors of Benign/Malignant from brain tumors knowledge base training system of deep learning.

\section{EXPERIMENT RESULTS}

Figure 5. a-b - illustrates input image type one. $\mathrm{c}$ - illustrates final combined image

Figure 5a and Figure 5c illustrates input image type one i.e. T1 weighted image and T2 weighted images. And Figure 5c illustrates final combined image. The quality of fused image can be verified by calculating some mathematical formulas such as PSNR, entropy, stander deviation and RMSE. These calculations for images in the dataset are given here in table. PSNR in the range of 42.83 values indicates that the fusion done is of best efficiency. Table 1 shows the performance parameters with its evaluation readings of various fused images

Table 2 shows the comparisons levels of various Fusion Techniques Performances evaluation from fusion of T1 and T2 Weighted MRI for tumor detection 
Figure 4. Architecture of training and testing using CLBP and PHOG

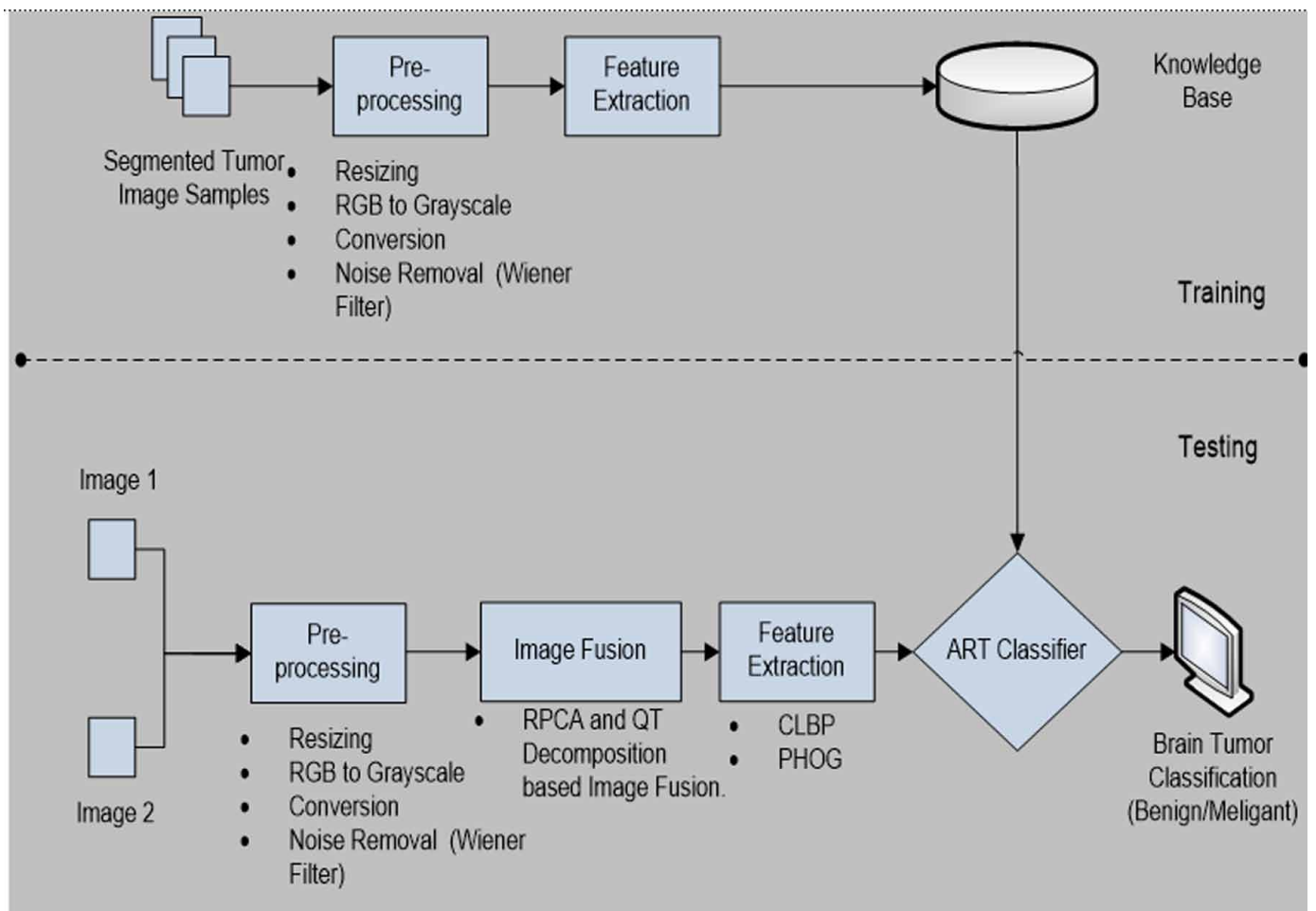

Table 1. Performance Evaluation of Fused images

\begin{tabular}{|c|c|c|c|c|}
\hline $\begin{array}{c}\text { Performance } \\
\text { Parameter }\end{array}$ & $\begin{array}{c}\text { Fused } \\
\text { Image 1 }\end{array}$ & $\begin{array}{c}\text { Fused } \\
\text { Image 2 }\end{array}$ & $\begin{array}{c}\text { Fused } \\
\text { Image 3 }\end{array}$ & $\begin{array}{c}\text { Fused } \\
\text { Image 4 }\end{array}$ \\
\hline PSNR & 42.83 & 42.95 & 42.89 & 42.85 \\
\hline RMSE & 2.2242 & 2.2063 & 2.2189 & 2.2173 \\
\hline ENTROPY & 7.0677 & 7.0833 & 7.0898 & 7.0983 \\
\hline $\begin{array}{c}\text { Standard } \\
\text { Deviation }\end{array}$ & 81.50 & 81.44 & 81.36 & 81.24 \\
\hline
\end{tabular}

\section{CONCLUSION}

The efficient tumor segmentation and identification is the objective of research work. Effectiveness of results is improved by fusing the brightness information of T1 and T2 images by applying fusion technique. Fused data have provided the needful for accurate segmentation. Level Set based segmentation has given a proper boundary based segmentation of tumor section. We are capable to get better PSNR as the fusion method is involved in initial stage.

With segmentation, classification of tumor part is major objective of research work. For this purpose feature vector calculation is made using the algorithms GLCM and GLRLM. The classification is completed in two levels; tumor non-tumor and benign or malignant. Based on application methodologies are varied and the effective results are obtained. PHOG and CLBP techniques stood better for the benign or malignant classification with ART classifier. The RPCA and Qudtree based 
Table 2. Performance results of various Fusion Techniques

\begin{tabular}{|c|c|c|}
\hline Sl.No & Fusion Techniques & Performance \\
\hline \multirow{2}{*}{1} & \multirow{2}{*}{ Most Dominant Features of Wavelet and Curvelet Transform techniques } & PSNR $=+42.66 \mathrm{~dB}$ \\
\hline & & $\mathrm{RMSE}=2.2767$ \\
\hline \multirow{4}{*}{2} & \multirow{4}{*}{$\begin{array}{l}\text { coefficients generated by RPCA (Robust Principal Component Analysis) } \\
\text { and Quadtree based decomposition techniques using CT and DWT }\end{array}$} & $\mathrm{PSNR}=24.95$ \\
\hline & & $\mathrm{RMSE}=2.2063$ \\
\hline & & Entropy $=7.0833$ \\
\hline & & Standard Deviation $=81.44$ \\
\hline 3 & $\begin{array}{l}\text { Tumour features are extracted using GLCM (Gray Scale Co-occurrence } \\
\text { Matrix) and GLRLM (Grey-level run-length matrix). }\end{array}$ & Accuracy $=93.45 \%$ \\
\hline 4 & $\begin{array}{l}\text { Tumour features are extracted using Complete Local Binary Pattern } \\
\text { (CLBP) and Pyramid Histogram of Orientation Gradients (PHOG). }\end{array}$ & Accuracy $=97.3 \%$. \\
\hline
\end{tabular}

decomposing with this final set of techniques have reached the accuracy level of $97.3 \%$. The collective performance of research work is classifying the dataset into tumor or non-tumor initially and finally based on pixels affected under tumor section sub classification i.e. benign or malignant classification is done and affected section is highlighted.

The future scope for the method can be analysing the levels of lesion in the section and producing the detailed information on sub levels of benign and malignant. 


\section{REFERENCES}

Babu \& Begum. (2015). An Adaptive MRI Tumour Detection Using Neural Network Based Adaboost Algorithm. International Journal of Computer Science and Information Technologies, 6(1), 42-47.

Candes \& Donoho. (2005). A Fast Discrete Curvelets Transform. Applied and Computational Mathematics, California Institute of Technology.

Candes, E. J., \& Donoho, D. L. (2000). Curvelets: A surprisingly effective nonadaptive representation for objects with edges. Vanderbilt University Press.

Charfi, S., Lahmyed, R., \& Rangarajan, L. (2014). A Novel Approach For Brain Tumor Detection Using Neural Network. International Journal of Research in Engineering \& Technology, 2(7), 93-104.

Jaffar, Mirza, \& Mahmud. (2011). MR imaging enhancement and segmentation of tumor using fuzzy curvelet. King Saud University.

Juan, E. (2010). Gender Classification from Iris Images using Fusion of Uniform Local Binary Patterns. Springer.

Mahantesh, \& Kanyakumari. (2016). BraTS: Brain Tumour Segmentation - Some Contemporary Approaches. International Journal of Innovative Research in Science, 5 (10).

Mustaqeem, A., Javed, A., \& Fatima, T. (2012). An Efficient Brain Tumour Detection Algorithm Using Watershed $\&$ Thresholding Based Segmentation. I.J. Image. Graphics and Signal Processing, 10(10), 34-39. doi:10.5815/ ijigsp.2012.10.05

Patil \& Mudengudi. (2011). Image fusion using Hierarchical PCA. In Image Information Processing (ICIIP), International Conference. IEEE.

Prakash, R., \& Mouli. (2012). Medical image fusion based on redundancy DWT and Mamdani type min-sum mean-of-max techniques with quantitative analysis. In Recent Advances in Computing and Software Systems (RACSS), International Conference. IEEE.

Samriti \& Singh. (2018). Brain Tumor Detection using Image Segmentation. International Journal of Engineering Development and Research, 4(2). http://www.ripublication.com

Sheela \& Babu. (2016). Analysis and Evaluation of Brain Tumour Detection from MRI Using F-PSO and FB-K Means. International Journal of Computer Science and Information Technology \& Security, 6(1).

Sinha, A., Banerji, S., \& Liu, C. (2012). Novel Color Gabor-LBP-PHOG (GLP) Descriptors for Object and Scene Image Classification. ICVGIP '12. doi:10.1145/2425333.2425391

Sruthy, P., \& Sasi. (2013). Image Fusion Technique using DT-CWT. In Automation, Computing, Communication, Control and Compressed Sensing (iMac4s), International Conference. IEEE.

Starck, J. L., Candes, E. J., \& Donoho, D. L. (2002). The Curvelet Transform for image denosing. IEEE Transactions on Image Processing, 11(6), 670-684. doi:10.1109/TIP.2002.1014998 PMID:18244665

Wright, J., Peng, Y., Ma, Y., Ganesh, A., \& Rao, S. (2009). Robust Principal Component Analysis: Exact Recovery of Corrupted Low-Rank Matrices via Convex Optimization. Advances in Neural Information Processing Systems.

Zaveri, T., Zaveri, M., Shah, V., \& Patel, N. (2009). A Novel Region Based Multifocus Image Fusion Method. Digital Image Processing, International Conference, 50-54. 